https://doi.org/10.22319/rmcp.v9i3.4358

Artículo

\title{
Metales pesados en leche de vacas alimentadas con alfalfa producida en suelos irrigados con aguas residuales en Puebla y Tlaxcala, México
}

\section{Heavy metals in milk from cows fed alfalfa produced in soils irrigated with wastewater in Puebla and Tlaxcala, Mexico}

Numa Pompilio Castro-González ${ }^{\text {ab* }}$

Rafael Moreno-Rojas ${ }^{\mathrm{b}}$

Francisco Calderón-Sánchez ${ }^{\mathrm{c}}$

Alicia Moreno-Ortegab

José Víctor Tamariz-Flores ${ }^{\mathrm{d}}$

a Facultad de Ingeniería Agrohidráulica. Benemérita Universidad Autónoma de Puebla, Av. Reforma 167, 73900, Tlatlauquitepec, Puebla, México.

b Departamento de Bromatología y Biotecnología de alimentos, Universidad de Córdoba. Córdoba, España.

${ }^{\mathrm{c}}$ Colegio de Postgraduados Campus-Puebla. Puebla, México.

${ }^{\mathrm{d}}$ Departamento de Investigación en Ciencias Agrícolas. Benemérita Universidad Autónoma de Puebla. Puebla, México.

* Autor de correspondencia: numa.castro@correo.buap.mx

\section{- Resumen:}

El objetivo fue determinar la presencia de $\mathrm{Cd}, \mathrm{Pb}, \mathrm{Ni}, \mathrm{Cu}, \mathrm{Cr}, \mathrm{Zn} \mathrm{y} \mathrm{As} \mathrm{en} \mathrm{la} \mathrm{cadena}$ alimentaria de la leche de vaca, producida en zonas donde la alfalfa es cultivada en suelos irrigados con aguas residuales de procedencia industrial, doméstica y agrícola. Se muestrearon suelo y alfalfa de 16 sitios ubicados en cuatro zonas; la leche se colectó de 160 
vacas, correspondiendo a 40 vacas de cuatro hatos diferentes por zona, todo en dos épocas del año y por triplicado. Se calculó el factor de bioacumulación (BCF), el factor de translocación (TF) y el valor de transferencia de los metales de la planta a la leche. Las plantas tuvieron un $\mathrm{BCF}<1$, indicando que la alfalfa es resistente a los metales pesados. Sin embargo, el TF >1 en orden decreciente quedó de la siguiente manera: $\mathrm{Zn}$; $\mathrm{Cu}$; $\mathrm{Ni}$; $\mathrm{Pb}$ y $\mathrm{Cr}$, lo que muestra la existencia de gran movilidad de los metales dentro de la planta. La leche tuvo un contenido de $\mathrm{Pb}$ en un rango de $0.039 \pm 0.02$ a $0.059 \pm 0.05 \mathrm{mg} \mathrm{kg}^{-1}$, valores por arriba del límite internacional permitido. Sin embargo, los niveles de $\mathrm{Pb}$ y As fueron inferiores a los valores permisibles por la Norma Oficial Mexicana. Se concluye que la alfalfa es una planta acumuladora y resistente a los metales pesados, y cuando es cultivada en suelos contaminados, se convierte en un medio importante para la trasferencia de metales pesados a los animales y son eliminados a través de la leche.

- Palabras clave: Bioacumulación, Translocación, Metales pesados, Contaminación alimentaria, Leche.

\section{- Abstract:}

The objective was to determine the presence of $\mathrm{Cd}, \mathrm{Pb}, \mathrm{Ni}, \mathrm{Cu}, \mathrm{Cr}, \mathrm{Zn}$ and $\mathrm{As}$ in the food chain of cow's milk, produced in areas where alfalfa is cultivated in irrigated soils with industrial, domestic and agricultural wastewater. Soil and alfalfa were sampled from 16 sites located in four zones; milk was collected from 160 cows, corresponding to 40 cows from four different herds per zone, all in two seasons of the year and in triplicate. The bioaccumulation factor (BCF), the translocation factor (TF) and the transfer value of the metals from the plant to the milk were calculated. The plants had a $\mathrm{BCF}<1$, indicating that alfalfa is resistant to heavy metals. However, the TF $>1$ in decreasing order was left as follows: $\mathrm{Zn} ; \mathrm{Cu} ; \mathrm{Ni} ; \mathrm{Pb}$ and $\mathrm{Cr}$, which shows the existence of great mobility of metals within the plant. The milk had a $\mathrm{Pb}$ content in a range of $0.039 \pm 0.02$ to $0.059 \pm 0.05 \mathrm{mg} \mathrm{kg}^{-1}$, values above the international allowed limit. However, the levels of $\mathrm{Pb}$ and $\mathrm{As}$ were lower than the permissible values by the Official Mexican Standard. It is concluded that alfalfa is an accumulator plant and resistant to heavy metals and when it is cultivated in contaminated soils, it becomes an important medium for the transfer of heavy metals to animals and they are eliminated through milk.

- Key words: Bioaccumulation, Translocation, Heavy metals, Food contamination, Milk.

Recibido 29/01/2017

Aceptado 19/12/2017 


\section{Introducción}

Los forrajes cultivados en suelos contaminados con metales pesados pueden traer consecuencias graves de salud y seguridad alimentaria. La contaminación ambiental por metales pesados producida por la industria y el crecimiento urbano afecta el aire, agua y suelos $^{(1)}$; pudiéndose acumular a largo plazo a niveles tóxicos en los suelos, donde permanece por largos periodos debido a que no son degradados y pueden biotransformarse en la cadena alimentaria $^{(2,3)}$. Esta acumulación puede provocar graves trastornos de salud en la población humana ${ }^{(4,5,6)}$. Los metales pesados y el As son acumulados en órganos importantes del cuerpo humano y el de los animales cuando son ingeridos en alimentos y agua de bebida ${ }^{(7,8,9)}$.

La alfalfa (Medicago sativa) es considerada como planta acumuladora con buen efecto remediador $^{(10,11)}$. Es utilizada para la alimentación de bovinos lecheros, debido a que es una leguminosa de gran aporte nutricional ${ }^{(12,13)}$. Por tanto esta leguminosa al ser cultivada en suelos irrigados con aguas procedentes de los ríos Atoyac, Zahuapan y la presa Manuel Ávila Camacho que reciben las descargas de las diferentes industrias ubicadas en los estados de Puebla y Tlaxcala, podrían contener metales pesados, los cuales pueden ser biotransformados a los animales que la consumen, originando acumulación en algunos órganos, y en parte ser excretados a través de la leche, poniendo en riesgo de salud a las personas que la consumen. Por tales razones, el objetivo de este trabajo fue determinar los factores de bioacumulación y translocación en alfalfa (Medicago sativa), así como el contenido $\mathrm{Cd}, \mathrm{Pb}, \mathrm{Ni}, \mathrm{Cu}, \mathrm{Cr}, \mathrm{Zn}$ and As en la leche de vacas alimentadas con alfalfa producida en suelos agrícolas de cuatro zonas irrigadas con aguas residuales en dos épocas del año; así como determinar los valores de biotransferencia de dichos metales de la alfalfa a la leche.

\section{Material y métodos}

\section{- Área de estudio •}

Se consideraron suelos y plantas de áreas que son irrigadas con aguas residuales procedentes de la industria, retornos agrícolas y de los hogares, así como la leche de las vacas que consumen dichos forrajes. Por ello se muestrearon cuatro zonas (Figura 1); la zona 1 
correspondió al municipio de Tepetitla de Lardizábal, la zona 2 a Nativitas (en ambas se aprovecha el río Atoyac), la zona 3 en Santa Isabel Tetlatlahuca irrigada por el río Zahuapan; estas tres zonas pertenecen al estado de Tlaxcala y la zona 4 en Tecamachalco, Puebla, donde se riega con aguas de la presa Manuel Ávila Camacho (canal de Valsequillo).

Figura 1: Localización de los sitios de muestreo de suelo, alfalfa y leche en las áreas irrigadas con aguas residuales en el estado de Puebla y Tlaxcala, México

\section{ÁREA DE MUESTREO}
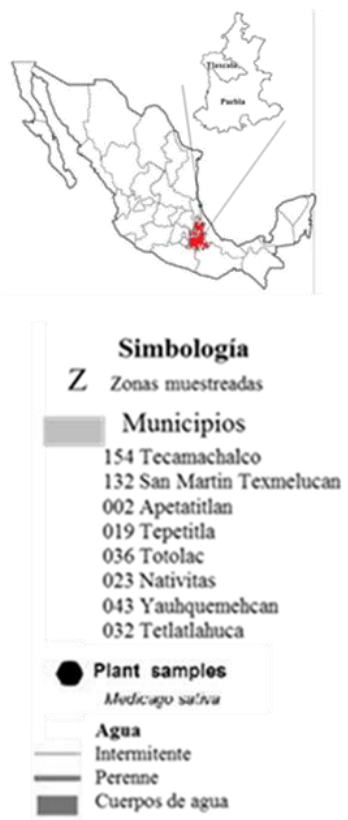

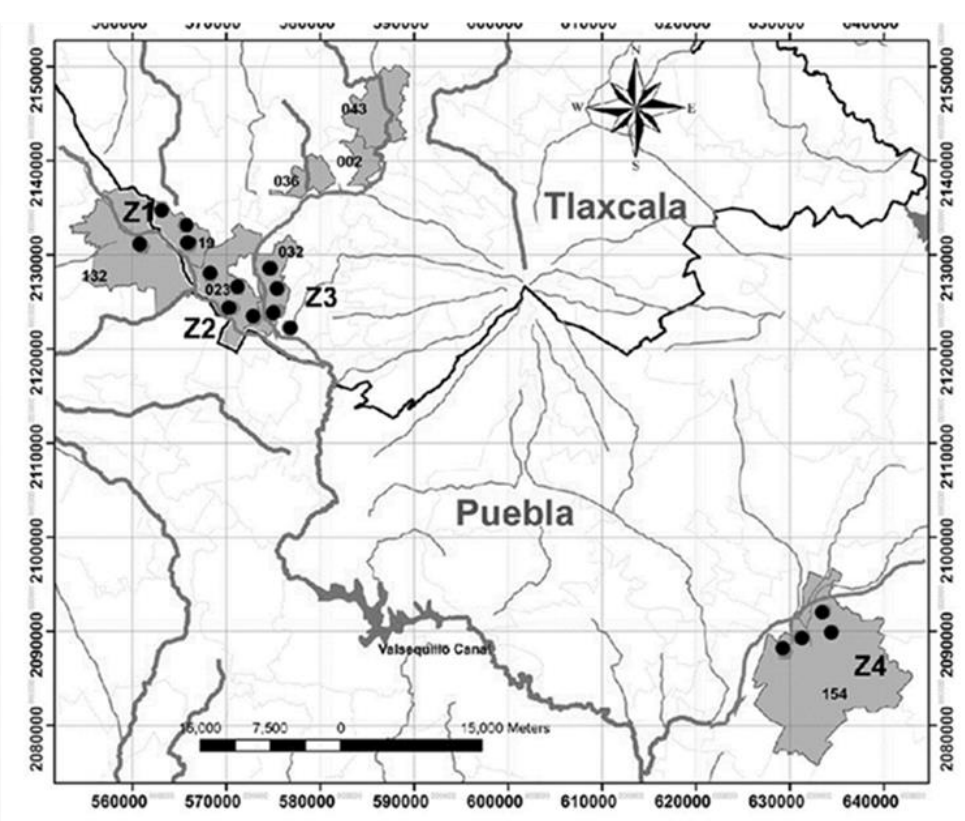

En las zonas de muestreo prevalece un clima templado húmedo con lluvias en verano, donde las principales actividades agropecuarias son la siembra de maíz y alfalfa, asociados a la producción de bovinos lecheros. Se localiza en la porción central del estado de Tlaxcala entre los paralelos $19^{\circ} 06^{\prime}$ y $19^{\circ} 40^{\prime} \mathrm{N}$ y $97^{\circ} 58^{\prime}$ y $98^{\circ} 03 \mathrm{O}$; cubre total o parcialmente 50 de los 60 municipios, representando el $52 \%$ de la superficie total del estado. Por otra parte, Tecamachalco, Puebla que se ubica en los paralelos $18^{\circ} 52^{\prime} 57^{\prime \prime} \mathrm{N}$ y a $97^{\circ} 43^{\prime} 49^{\prime \prime} \mathrm{O}^{(14,15)}$. 


\section{- Muestreo de suelo, alfalfa y leche •}

El muestreo se realizó en dos épocas del año, comprendidas en julio 2014 (verano) y marzo de 2015 (primavera). Las muestras de suelo se recolectaron en cuatro zonas definidas por la fuente del agua de riego, formando un total de 16 sitios, correspondientes a cuatro muestras por zona (Figura 1). Las muestras se tomaron de la capa superficial del suelo $(0$ a $30 \mathrm{~cm}$ ) debido a que los metales en los suelos cultivados se encuentran distribuidos de manera homogénea a este nivel, como consecuencia de la aplicación de abonos, fertilizantes y el contenido de materia orgánica del suelo ${ }^{(16)}$. Se colectaron 10 sub muestras en cada sitio, las cuales se mezclaron para formar una muestra compuesta de cada sitio. Estas se colocaron en bolsas de polietileno negro, que se cerraron evitando la presencia de aire, y llevadas al laboratorio, donde se secaron en bandeja de papel, alcanzando una profundidad máxima de $2.5 \mathrm{~cm}$, una temperatura no superior a $35^{\circ} \mathrm{C}$ y una humedad relativa entre 30 y $70 \%$ a la sombra durante ocho días, con el fin de hacer un mejor manejo y homogeneización de las muestras. Se removieron las rocas y material orgánico, luego las muestras se trituraron utilizando un mazo de madera, y se realizó el tamizado en un tamiz de nylon de $<2 \mathrm{~mm}$ de diámetro, y se conservaron en bolsas de nylon perfectamente cerradas hasta su digestión de acuerdo con la norma NOM-021-SEMARNAT-2000 ${ }^{(17)}$.

La alfalfa utilizada se colectó por triplicado al inicio de la floración, de los mismos sitios de donde se extrajo el suelo, a una profundidad de 0 a $30 \mathrm{~cm}^{(18)}$ y se trasportaron en bolsas oscuras al laboratorio, donde se les lavó con agua desionizada hasta eliminar todo tipo de residuos de suelo, al mismo tiempo que fueron pesadas en su totalidad y por partes, siendo separadas en parte comestible (hoja-tallo) y raíz ${ }^{(19,20)}$, congelándolas para luego ser liofilizadas. Posterior a ello se molieron, tamizaron y almacenaron en bolsas de nylon hasta su digestión.

La leche se colectó de 160 vacas, correspondiendo a 40 vacas de cuatro hatos diferentes por zona, todas alimentadas con alfalfa cultivada en los suelos muestreados. Para ello se utilizaron tubos Falcón de $50 \mathrm{ml}$, previamente lavados con $\mathrm{HNO}_{3}$ al $10 \%$ v/v y fueron enjuagados por tres veces con agua desionizada para eliminar los residuos ácidos y evitar alteraciones en la leche. El muestreo se realizó una sola vez por la mañana al inicio del ordeño, directamente de la ubre, transportándolas en neveras al laboratorio, donde se congelaron a $-65^{\circ} \mathrm{C}$ y posteriormente liofilizadas. Para la liofilización de la alfalfa y leche se utilizó un equipo de liofilización LABCONCO Freezone de 4,5 litros (Kansas City, Missouri). 


\section{- Determinación de metales pesados}

Todas las muestras de suelo, planta y leche fueron previamente digeridas en un horno de microondas (CEM-MarsX, CEM corporation Mathews, Notrh Carolina). Para el suelo, se realizó una digestión bajo el protocolo EPA 3051, pesando $0.5 \mathrm{~g}$ de suelo y se añadieron 10 $\mathrm{ml}$ de ácido nítrico $\left(\mathrm{HNO}_{3}\right)$, se utilizó una potencia de $1,600 \mathrm{~W}$, con una rampa de $5 \mathrm{~min}$, presión de $350 \mathrm{psi}$, temperatura de $175^{\circ} \mathrm{C}$ y un intervalo de $5 \mathrm{~min}$.

Para la alfalfa se utilizó la parte comestible y la raíz, de cada uno, y por separado se pesaron $0.3 \mathrm{~g}$ y se agregaron $5 \mathrm{ml}$ de $\mathrm{HNO}_{3}$ más $5 \mathrm{ml}$ de peróxido de hidrógeno al $30 \% w / v$ a 1,600 $\mathrm{W}$ de potencia, con un tiempo de rampa de $15 \mathrm{~min}$, presión de $800 \mathrm{psi}, 200{ }^{\circ} \mathrm{C}$ de temperatura y $10 \mathrm{~min}$ de espera. La digestión de la leche liofilizada se realizó utilizando $0.5 \mathrm{~g}$, a la cual se le agregaron $10 \mathrm{ml}$ de $\mathrm{HNO}_{3}$, colocándola a una potencia de $1,600 \mathrm{~W}$, rampa de $5 \mathrm{~min}$, presión de $350 \mathrm{psi}$, temperatura de $175^{\circ} \mathrm{C}$ y un intervalo de $5 \mathrm{~min}$. Una vez digeridas cada una de las muestras se filtraron sobre papel Whatman grado 42 , se aforaron a $50 \mathrm{ml}$ con agua desionizada y se refrigeraron hasta su análisis. El ácido nítrico utilizado para cada digestión fue $\mathrm{HNO}_{3}$ High purity (65\%, Suprapur, Merck, Darmstadt, Germany).

Los metales pesados $\mathrm{Cd}, \mathrm{Pb}, \mathrm{Ni}, \mathrm{Cu}, \mathrm{Cr}, \mathrm{Zn}$ y As se determinaron por medio de espectroscopia de emisión óptica de plasma acoplado inductivamente (ICP - OES, Varian 730 - ES). Todos los productos químicos utilizados fueron de grado reactivo analítico. Se prepararon las soluciones en agua desionizada $18.2 \mathrm{M} \Omega \mathrm{cm}$. Los estándares de calibración para cada metal se prepararon utilizando solución patrón ICP multielemento standard XVI, compuesta de 21 elementos en $\mathrm{HNO}_{3}$ Suprapuro 6\%, con densidad de $1.032 \mathrm{~g} / \mathrm{cm}^{3}$ y $20{ }^{\circ} \mathrm{C}$ de Merck KGaA, Frankfurter Str. 250, 64293 Darmstadt, Germany.

Los niveles de precisión y exactitud se realizaron con cinco blancos y diez repeticiones. El valor de recuperación analítica se determinó en $106 \%$ en promedio, el coeficiente de correlación $\left(\mathrm{r}^{2}\right)$ fue 0.9999 para todos los metales. Los límites de detección y los límites de cuantificación (LOD - LOQ) se calcularon con 3 y 10 veces la desviación estándar del blanco. 


\section{- Factor de bioconcentración (BCF) y factor de translocación (TF)}

Con los valores de suelo y planta se realizaron los cálculos para determinar el factor de bioconcentración (BCF), que indica la capacidad o eficiencia que poseen las plantas para acumular metales pesados en la raíz con relación a la concentración de metales existente en el suelo o sustrato, calculándose en base a peso $\operatorname{seco}^{(21,22)}$; y el factor de translocación (TF) que indica la absorción de metales pesados que ejercen las plantas a los diferentes órganos vegetativos con respecto a la raíz ${ }^{(23,24,25)}$. Ambos factores se calcularon siguiendo las siguientes ecuaciones, las cuales han sido utilizadas por varios autores ${ }^{(26-29)}$ :

$$
B C F=\frac{C_{\text {raiz }}}{C_{\text {suelo }}} \quad T F=\frac{C_{\text {parte comestible }}}{C_{\text {raíz }}}
$$

Donde $B C F$ es el factor de bioconcentración, $T F$ es el factor de translocación y $C$ es la concentración del metal (suelo, raíz y parte aérea o comestible).

\section{- Análisis estadístico •}

La información obtenida de cada componente se analizó bajo un diseño completamente al azar con arreglo factorial, en el que los factores fueron: la estación del año y las zonas, utilizando un modelo lineal generalizado (GLM), y para la comparación de medias se efectuó la prueba de Tukey con el paquete estadístico SAS Ver. $9^{(30)}$.

Con los valores obtenidos del contenido de metales en la alfalfa y la leche se determinó el valor de biotransferencia de metales de la planta hacia los animales, utilizando la ecuación propuesta por Stevens ${ }^{(31)} ; B T F\left(\mathrm{~kg} \mathrm{día}^{-1}\right)=$ Concentración del metal en leche $\left(\mathrm{mg} \mathrm{kg}^{-1}\right) /$ Consumo promedio diario del metal ( $\left.m g d^{\prime i} a^{-1}\right)$; y para ello se consideró que son vacas de la raza Holstein, con un peso promedio de $600 \pm 34 \mathrm{~kg}$ y que tienen una ingesta de $10 \mathrm{~kg}$ de alfalfa al día en base seca. 


\section{Resultados y discusión}

\section{-Suelo}

Por zonas, el suelo agrícola mostró diferencias significativas $(P<0.01)$, para $\mathrm{Ni}$ y $\mathrm{Cr}$, siendo la zona 4 la que tuvo los valores más bajos (Cuadro 1). En cuanto a la época del año, no existió diferencia significativa en el contenido de metales en el suelo. Siebe ${ }^{(32)}$ en un trabajo realizado en suelos del estado de Hidalgo, irrigados con aguas residuales procedentes de la ciudad de México, reportó niveles de 24, 20, 52 y $77 \mathrm{mg} \mathrm{kg}^{-1}$ de $\mathrm{Pb}, \mathrm{Cu}, \mathrm{Cr}$ y $\mathrm{Zn}$ respectivamente, los cuales son superiores a los encontrados en este trabajo y para $\mathrm{Cd} 0.57$ mg kg-1 siendo un nivel más bajo que el detectado en esta investigación. Sin embargo, los suelos analizados por Siebe ${ }^{(32)}$ estuvieron irrigados por más de 80 años, a diferencia de los de este trabajo que tienen 40 años de irrigación con las aguas residuales de procedencia industrial.

Cuadro 1: Contenido de metales pesados por zona $\left(\mathrm{mg} \mathrm{kg}^{-1}\right)$ en suelos agrícolas irrigados con aguas residuales en Puebla y Tlaxcala, México

\begin{tabular}{lccccccc}
\hline Zona & $\mathrm{Cd}$ & $\mathrm{Pb}$ & $\mathrm{Ni}$ & $\mathrm{Cu}$ & $\mathrm{Cr}$ & $\mathrm{Zn}$ & $\mathrm{As}$ \\
\hline 1 & $1.16 \pm 0.2^{\mathrm{a}}$ & $14.79 \pm 1.2^{\mathrm{a}}$ & $15.40 \pm 1.5^{\mathrm{ab}}$ & $12.19 \pm 1.6^{\mathrm{a}}$ & $19.04 \pm 3.3^{\mathrm{ab}}$ & $25.33 \pm 1.5^{\mathrm{a}}$ & $5.16 \pm 1.1^{\mathrm{a}}$ \\
2 & $1.15 \pm 0.3^{\mathrm{a}}$ & $13.03 \pm 4.8^{\mathrm{a}}$ & $14.70 \pm 3.6^{\mathrm{ab}}$ & $11.24 \pm 3.1^{\mathrm{a}}$ & $17.35 \pm 3.2^{\mathrm{ab}}$ & $20.72 \pm 3.5^{\mathrm{a}}$ & $5.91 \pm 2.2^{\mathrm{a}}$ \\
3 & $1.42 \pm 0.5^{\mathrm{a}}$ & $17.67 \pm 7.1^{\mathrm{a}}$ & $16.69 \pm 4.6^{\mathrm{a}}$ & $14.20 \pm 4.7^{\mathrm{a}}$ & $20.25 \pm 5.8^{\mathrm{a}}$ & $25.23 \pm 4.9^{\mathrm{a}}$ & $5.70 \pm 1.2^{\mathrm{a}}$ \\
4 & $1.22 \pm 0.2^{\mathrm{a}}$ & $13.60 \pm 2.6^{\mathrm{a}}$ & $12.40 \pm 2.0^{\mathrm{b}}$ & $14.62 \pm 3.6^{\mathrm{a}}$ & $14.37 \pm 2.2^{\mathrm{b}}$ & $20.29 \pm 3.4^{\mathrm{a}}$ & $4.51 \pm 2.6^{\mathrm{a}}$ \\
\hline
\end{tabular}

ab Diferentes literales representan diferencias entre zonas $(P \leq 0.05)$.

\section{- Alfalfa}


El contenido de metales en la parte comestible (hoja - tallo) (Cuadro 2) no evidenció diferencia significativa entre zonas. Sin embargo, por época del año el $\mathrm{Pb}$, Ni y $\mathrm{Zn}$ fueron diferentes $(P<0.01)$ mostrando valores más altos en el verano, caso contrario para el $\mathrm{Cr}$ que tuvo nivel más alto en primavera.

Cuadro 2: Contenido de metales pesados ( $\mathrm{mg} \mathrm{kg}^{-1} \mathrm{MS}$ ) en alfalfa (raíz y parte comestible) por zona y época en zonas irrigadas con aguas residuales en Puebla y Tlaxcala, México

\begin{tabular}{|c|c|c|c|c|c|c|c|c|}
\hline & & $\mathrm{Cd}$ & $\mathrm{Pb}$ & $\mathrm{Ni}$ & $\mathrm{Cu}$ & $\mathrm{Cr}$ & $\mathrm{Zn}$ & As \\
\hline & Zona & & & & & & & \\
\hline \multirow{4}{*}{ Parte comestible } & 1 & $0.05 \pm 0.03^{a}$ & $1.86 \pm 1.0^{\mathrm{a}}$ & $3.06 \pm 2.0^{\mathrm{a}}$ & $2.39 \pm 0.9^{a}$ & $1.56 \pm 0.6^{a}$ & $10.68 \pm 4.6^{a}$ & $1.25 \pm 0.6^{\mathrm{a}}$ \\
\hline & 2 & $0.09 \pm 0.04^{a}$ & $2.04 \pm 0.4^{a}$ & $3.15 \pm 0.7^{a}$ & $3.74 \pm 1.9^{a}$ & $2.13 \pm 1.6^{a}$ & $14.20 \pm 4.3^{a}$ & $0.88 \pm 0.6^{a}$ \\
\hline & 3 & $0.07 \pm 0.05^{a}$ & $2.26 \pm 1.4^{a}$ & $4.36 \pm 3.3^{a}$ & $3.27 \pm 1.2^{\mathrm{a}}$ & $1.79 \pm 0.9^{a}$ & $15.26 \pm 7.7^{a}$ & $1.12 \pm 0.8^{\mathrm{a}}$ \\
\hline & 4 & $0.07 \pm 0.03^{a}$ & $1.96 \pm 1.3^{a}$ & $4.23 \pm 2.4^{a}$ & $3.40 \pm 1.3^{a}$ & $2.82 \pm 1.5^{\mathrm{a}}$ & $11.20 \pm 8.2^{a}$ & $0.80 \pm 1.5^{\mathrm{a}}$ \\
\hline \multicolumn{9}{|l|}{ Época } \\
\hline Verano & & $0.06 \pm 0.04^{a}$ & $2.69 \pm 0.9^{a}$ & $5.06 \pm 2.4^{a}$ & $2.66 \pm 1.2^{\mathrm{a}}$ & $1.36 \pm 0.9^{b}$ & $15.92 \pm 6.5^{\mathrm{a}}$ & $1.31 \pm 0.5^{\mathrm{a}}$ \\
\hline Primavera & & $0.08 \pm 0.04^{a}$ & $1.36 \pm 0.7^{b}$ & $2.34 \pm 0.9 \mathrm{~b}$ & $3.74 \pm 1.5^{\mathrm{a}}$ & $2.78 \pm 1.2^{\mathrm{a}}$ & $9.75 \pm 4.8^{b}$ & $0.71 \pm 0.7^{\mathrm{a}}$ \\
\hline \multirow{4}{*}{ Raíz } & 1 & $0.04 \pm 0.03^{a}$ & $1.42 \pm 0.1^{\mathrm{a}}$ & $2.04 \pm 1.2^{\mathrm{a}}$ & $1.40 \pm 0.4^{b}$ & $1.18 \pm 0.7^{b}$ & $3.90 \pm 1.6^{a}$ & $0.16 \pm 2.0^{\mathrm{b}}$ \\
\hline & 2 & $0.10 \pm 0.06^{a}$ & $1.53 \pm 0.1^{\mathrm{a}}$ & $3.97 \pm 2.2^{\mathrm{a}}$ & $2.90 \pm 1.3^{\mathrm{ab}}$ & $2.64 \pm 0.9 \mathrm{a}$ & $7.81 \pm 3.4^{a}$ & $0.62 \pm 0.9^{b}$ \\
\hline & 3 & $0.06 \pm 0.03^{a}$ & $2.07 \pm 2.1^{\mathrm{a}}$ & $3.22 \pm 1.5^{a}$ & $2.17 \pm 0.9^{\mathrm{ab}}$ & $1.28 \pm 0.7^{b}$ & $7.08 \pm 4.5^{\mathrm{a}}$ & $0.80 \pm 2.2^{b}$ \\
\hline & 4 & $0.13 \pm 0.1^{\mathrm{a}}$ & $2.67 \pm 2.2^{\mathrm{a}}$ & $2.82 \pm 0.7^{\mathrm{a}}$ & $4.06 \pm 0.9^{a}$ & $1.85 \pm 0.8^{\mathrm{ab}}$ & $9.27 \pm 4.7^{a}$ & $2.54 \pm 2.6^{\mathrm{a}}$ \\
\hline \multicolumn{9}{|l|}{ Época } \\
\hline Verano & & $0.09 \pm 0.08^{a}$ & $2.88 \pm 1.6^{a}$ & $3.85 \pm 1.7^{a}$ & $3.19 \pm 0.9^{a}$ & $1.34 \pm 0.9^{b}$ & $5.43 \pm 3.2^{\mathrm{a}}$ & $1.48 \pm 2.5^{\mathrm{a}}$ \\
\hline Primavera & & $0.08 \pm 0.06^{a}$ & $0.96 \pm 0.9^{b}$ & $2.17 \pm 0.9^{b}$ & $2.16 \pm 2.2^{\mathrm{a}}$ & $2.14 \pm 0.8^{\mathrm{a}}$ & $8.59 \pm 5.8^{a}$ & $0.19 \pm 0.3^{\mathrm{a}}$ \\
\hline
\end{tabular}

MS=Materia seca.

${ }^{\mathrm{ab}}$ Diferentes literales representan diferencias entre zonas $(P \leq 0.05)$.

En otras regiones de México, Vázquez-Alarcón et $a l^{(33)}$ reportaron un contenido de Cd de 0.7 a $2.7 \mathrm{mg} \mathrm{kg}^{-1}$ en alfalfa, superior a lo encontrado en esta investigación, y en el caso del $\mathrm{Pb}$ (1.2 a $1.7 \mathrm{mg} \mathrm{kg}^{-1}$ ), los valores son inferiores a los determinados en este trabajo. El contenido en la raíz de alfalfa indica que existieron diferencias $(P<0.01)$, siendo As, $\mathrm{Cu}$ en la zona 4 y $\mathrm{Cr}$ en la zona 2 los que obtuvieron los mayores valores. Al comparar entre componentes (parte comestible y raíz) únicamente se obtuvo diferencia significativa $(P<0.01)$ para el contenido de $\mathrm{Zn}\left(12.09 \pm 5.5 \mathrm{mg} \mathrm{kg}^{-1}\right)$ en la parte comestible, siendo mayor que el detectado en la raíz $\left(7.15 \pm 5.2 \mathrm{mg} \mathrm{kg}^{-1}\right)$. El resto de metales no mostraron diferencias; esto podría deberse a que el año 2015 fue clasificado como un año de alta precipitación pluvial. Según CONAGUA $^{(34)}$, marzo contribuyó con el $7.9 \%$ a la hoja nacional de agua, equivalente a cuatro veces su contribución normal, de modo que en este mes, se produjo una precipitación 
de $125 \mathrm{~mm}$, a diferencia de 2014 que tuvo una precipitación de $25 \mathrm{~mm}$ en el mismo mes. Para julio de 2014, la precipitación fue $150 \mathrm{~mm}$.

En el caso particular del $\mathrm{Pb}$ al no haber diferencia entre raíz y la parte comestible, puede deberse a que la vía principal de contaminación con el $\mathrm{Pb}$ no es la raíz, aunque tiende a ser acumulado en ésta, debido a que el $\mathrm{Pb}$ es poco soluble ${ }^{(35,36)}$, lo cual contribuye a que posea menor movilidad, denotando que este elemento también puede estar ingresando a nivel de las hojas.

Aunque las plantas de alfalfa tienen gran capacidad acumuladora de metales pesados ${ }^{(37,38)}$; existen diferentes mecanismos de absorción y factores que pueden influir a que los metales no sean movilizados o no estén biodisponibles. Los metales se transportan a la raíz por flujo de agua en forma de iones, los cuales se absorben por estar en contacto con las raíces cuando se encuentran solubles en agua. La baja movilización del $\mathrm{Pb}$ puede deberse a la capacidad que tiene la planta de excluir el metal del paso de la raíz al suelo, o bloqueando el movimiento del metal dentro de la planta por medios péptidos (fitoquelatinas), aminoácidos (histidina y cisteína), compuestos fenólicos y ácidos orgánicos (cítrico, oxálico y málico), que secuestran o forman compuestos mediante la unión de ligandos específicos; además del valor del pH y las condiciones de textura y materia orgánica presentes en los suelos ${ }^{(39)}$, lo que puede contribuir a que exista menor solubilidad impidiendo la movilización.

Por tanto, la presencia del $\mathrm{Pb}$ y As en hojas puede deberse a que han sido absorbidos a nivel de las hojas debido a contaminación aérea. Otro mecanismo de absorción de metales por las plantas es la difusión que ocurre por gradiente de concentración de mayor a menor. También la intersección de la raíz con el suelo, la cual depende del tamaño, profundidad y el agua libre del suelo, que puede ser absorbida del espacio entre las partículas del suelo, siendo aprovechada esta vía tanto para los nutrientes como por los metales pesados ${ }^{(40)}$. Además, se ha mencionado que las sinergias y antagonismos que pueden existir entre los metales conducen a una mayor absorción, que puede ser benéfica o perjudicial al crecimiento de las

plantas, y con ello la acumulación en la misma ${ }^{(41)}$. Tal es el caso del $\mathrm{Pb}$ y $\mathrm{Cd}$, que de manera individual ocasionan daños negativos en las plantas, pero cuando aparecen juntos dentro de ésta, la fitotoxicidad se ve aumentada.

\section{- Factor de bioconcentración (BCF) y factor de translocación (TF)}

Los valores de BCF (Figura 2) indican que las plantas de alfalfa en las cuatro zonas tuvieron un valor inferior a uno para todos los metales estudiados. En el análisis del BCF por épocas 
se encontró que existió diferencia significativa $(P<0.01)$, donde los valores de $\mathrm{Pb}$, Ni y As fueron superiores en el verano (Figura 3) y el Zn mostró su nivel más alto en primavera. Así se tiene que los metales menos disponibles en orden ascendente fueron el $\mathrm{Cd}, \mathrm{Cr}, \mathrm{Pb}$. $\mathrm{Sin}$ embargo, para ningún metal y en ninguna de las dos estaciones climáticas muestreadas existieron valores $\mathrm{BCF}>1$.

Figura 2: Factor de bioconcentración $(\mathrm{BCF})$ de metales pesados en alfalfa cultivada en áreas irrigadas con aguas residuales

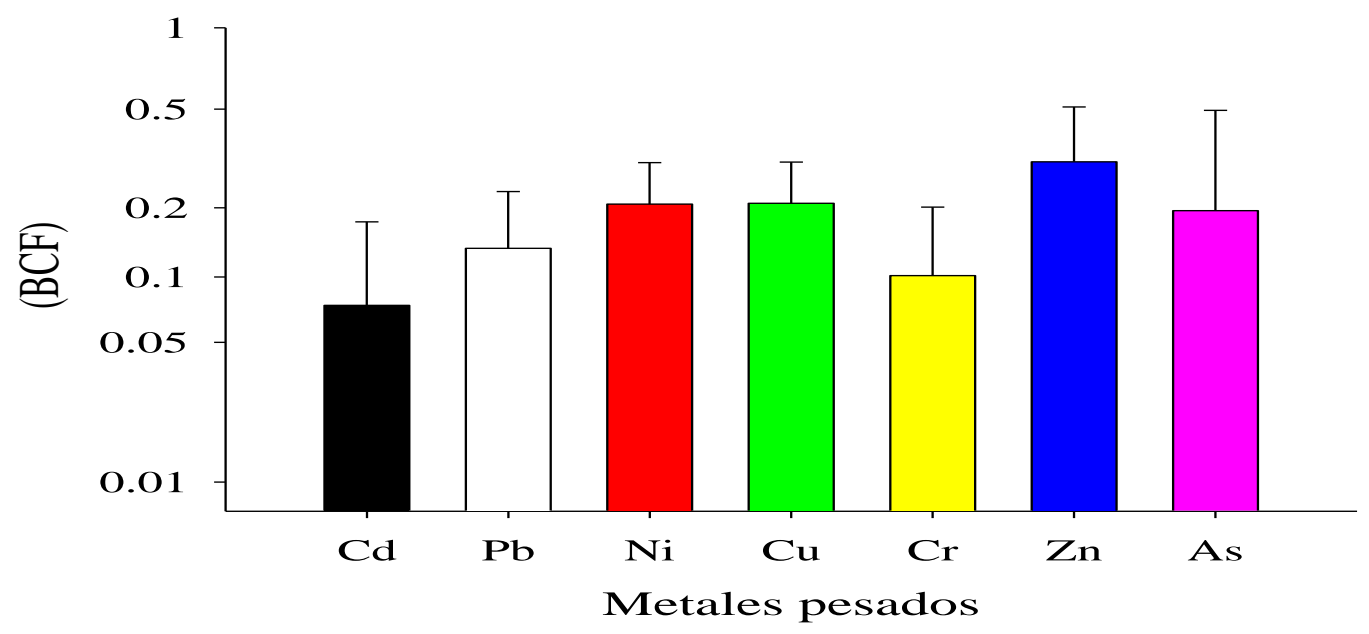

Los datos son medias y la línea vertical representa la desviación estándar.

Figura 3: Factor de bioconcentración (BCF) por época del año en alfalfa cultivada en áreas irrigadas con aguas residuales

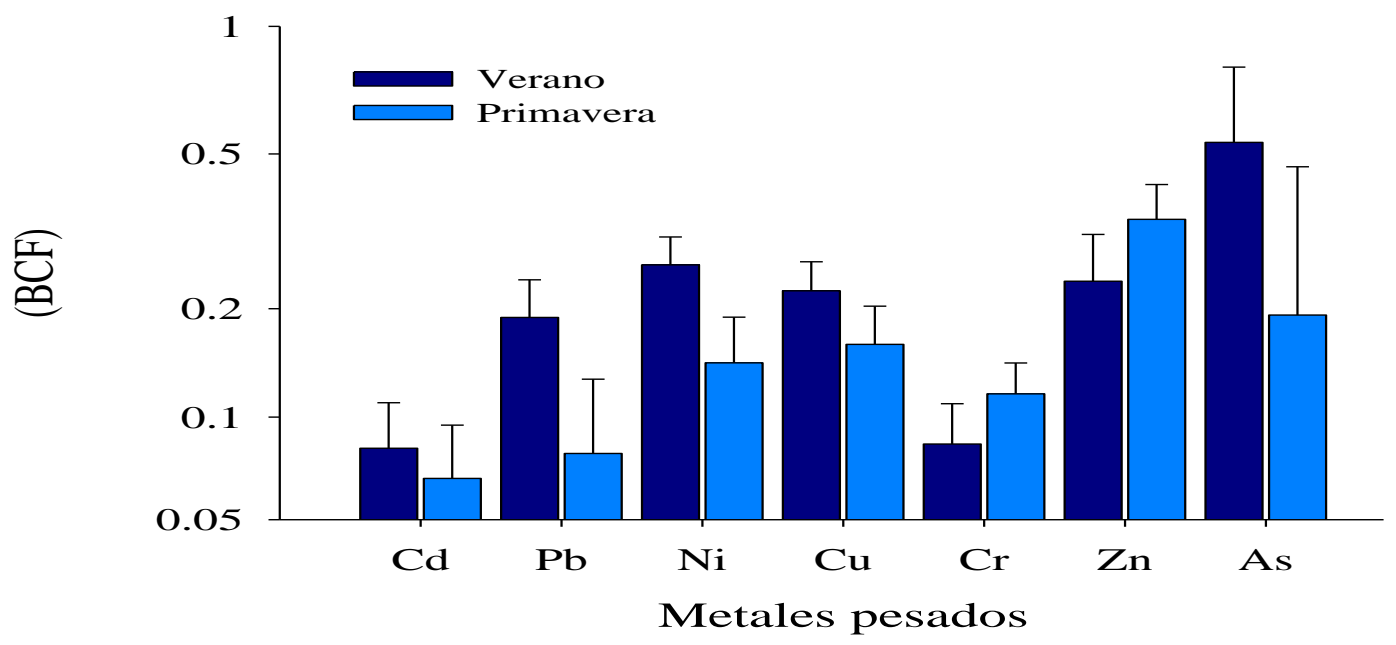

Los datos son medias y la línea vertical representa la desviación estándar. 
Algunos autores ${ }^{(42,43)}$ mencionan que cuando $\mathrm{TF}>1$, se considera que las plantas son acumuladoras de metales pesados, y en el presente estudio los valores encontrados (Figura 4) demuestran que en orden decreciente los metales que mostraron mayor movilidad de la raíz a la parte aérea de la planta fueron; $\mathrm{Zn}, \mathrm{Cu}, \mathrm{Ni}, \mathrm{Pb}$ y $\mathrm{Cr}$. En el caso del $\mathrm{Cd}$, estuvo en el límite y el As es el metal con la menor movilidad presentada en esta investigación.

Figura 4: Factor de translocación (TF) de metales pesados en alfalfa irrigada con aguas residuales

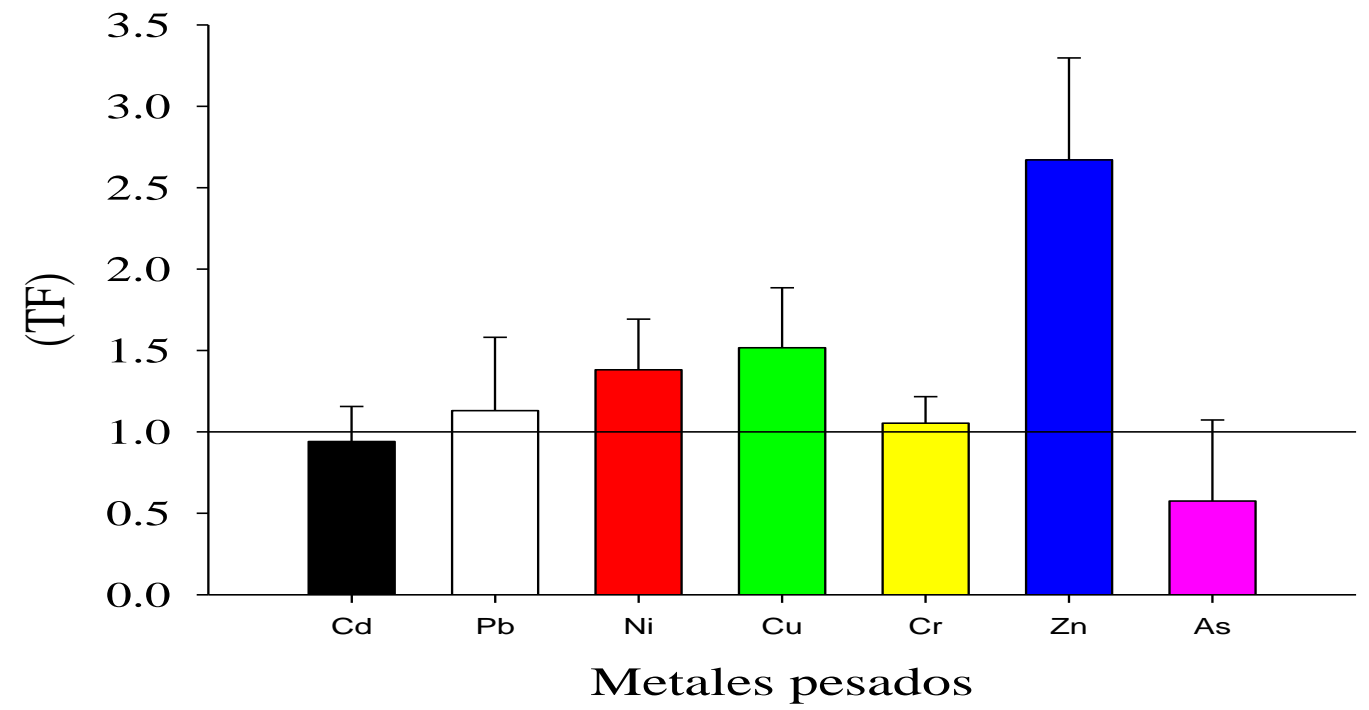

Los datos son medias y la línea vertical representa la desviación estándar.

En consecuencia, se considera que las alfalfas muestreadas en este trabajo tienen características de ser acumuladoras de metales pesados. Los valores obtenidos al evaluar el $\mathrm{TF}$ (Figura 5) durante las épocas demuestran diferencia significativa $(P<0.01)$, siendo en verano donde se detectó mayor movilidad de $\mathrm{Pb}, \mathrm{Ni}, \mathrm{Cu}$ y $\mathrm{Zn}$. En el caso de $\mathrm{Cr}$ la movilidad fue mayor en primavera. El Cd presentó valores que están en los límites, y se le considera con buena movilidad por la importancia tóxica que representa, y por ser altamente soluble en agua $^{(44)}$. 
Figura 5: Factor de translocación (TF) de metales pesados por época del año en alfalfa irrigada con aguas residuales

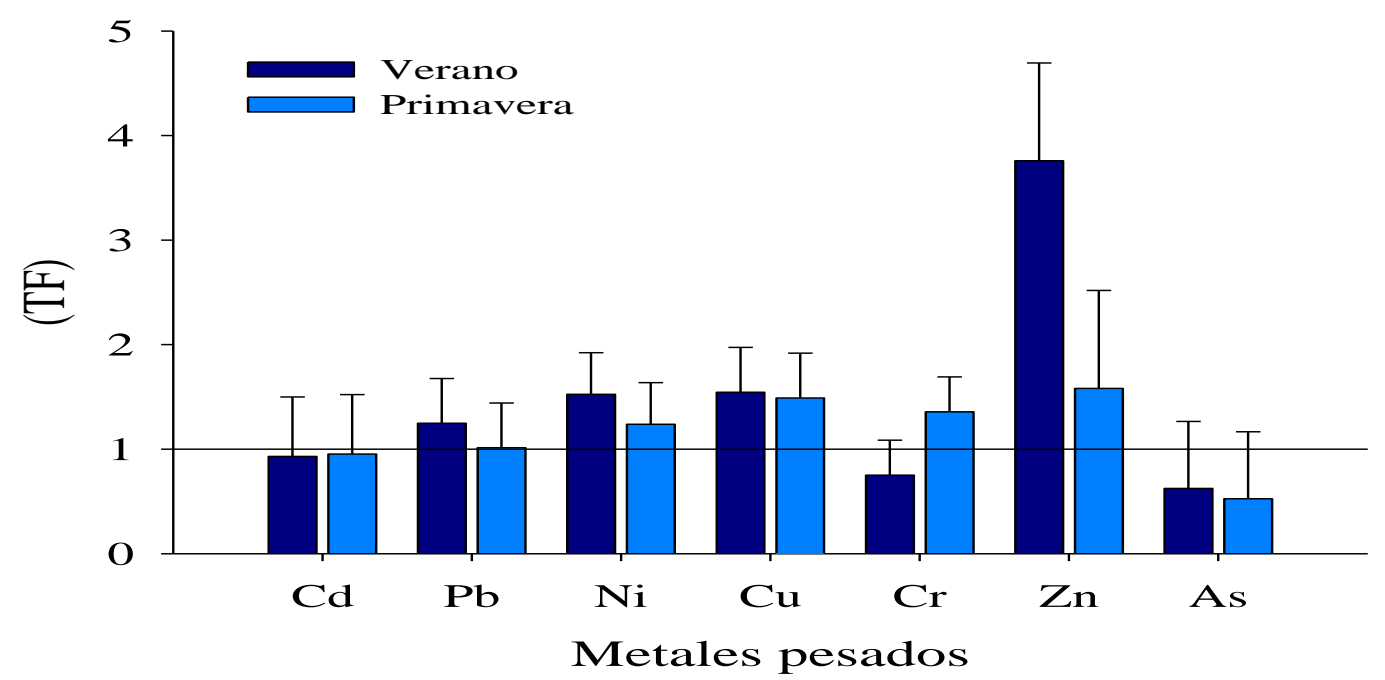

Los datos son medias y la línea vertical representa la desviación estándar.

Siendo la alfalfa una planta considerada como acumuladora de metales pesados, hace suponer un riesgo de contaminación por la ingestión que de ella realizan los animales ${ }^{(45)}$. Algunos metales como el $\mathrm{Co}, \mathrm{Mn}, \mathrm{Fe}, \mathrm{Se}, \mathrm{Zn}, \mathrm{Cu}$ y Ni, son esenciales para las funciones biológicas y fisiológicas de plantas, incluyendo la biosíntesis de proteínas, ácidos nucleicos, sustancias de crecimiento, síntesis de clorofila entre otras funciones; pero más allá de los límites permisibles, estos elementos metálicos se vuelven tóxicos dependiendo de la naturaleza, especies de metal y plantas $^{(46)}$.

Aunque las plantas tienen un mecanismo de desintoxicación efectuado por las fitoquelatinas, éstas no logran evitar la captación por parte de los rumiantes, ya que cuando los metales están unidos a las fitoquelatinas, al ser ingeridas por el animal forman un compuesto de glutamilcisteína en el rumen y el intestino delgado, pero es probable que este compuesto sea degradado rápidamente por los microorganismos del rumen, liberando así al metal que tiende a ser acumulado en los tejidos animales ${ }^{(47)}$. Principalmente en el caso de aquellos metales que no son necesarios para el metabolismo de los animales y que son considerados como de mayor toxicidad $(\mathrm{Cd}, \mathrm{Pb})$; y en el caso del $\mathrm{As}$, se le ha considerado con alguna función en el metabolismo de plantas y animales; sin embargo, es un metaloide sumamente tóxico, y se les considera a los tres como cancerígenos.

No obstante los valores encontrados en la parte comestible de la alfalfa, son bajos comparados con las normas establecidas para límites permisibles de metales en alimentos para ganado; pero hay que considerar que dichos límites han sido instaurados sin ser objetivos $^{(47)}$, por lo consiguiente la acumulación de los diferentes metales en rumiantes está relacionada más con el intervalo de tiempo de ingestión de los metales contenidos en los 
alimentos y la baja tasa de excreción de estos ${ }^{(47,48)}$. Los metales tóxicos pueden tener bajos índices de absorción, pero logran utilizar mecanismos de absorción supuestos para otros metales tales como el $\mathrm{Cd}-\mathrm{Zn}$ y $\mathrm{Pb}-\mathrm{Ca}$, lo que hace posible la contaminación de los productos y subproductos animales para consumo humano.

Así se tiene que el $\mathrm{Cd}$ se acumula principalmente en riñones e hígado y el $\mathrm{Pb}$ se deposita primordialmente en huesos largos y puede ser movilizado a la leche de las vacas principalmente después del parto ${ }^{(49,50)}$. La contaminación de los suelos y la edad de los animales son predictores de contaminación animal ${ }^{(51)}$, y los valores de los metales en suelo utilizados para la realización de esta investigación son indicativas de contaminación, aunado a la edad de las vacas, las cuales están por arriba de los tres años de edad, tiempo necesario para que exista acumulación de metales pesados en el cuerpo de los bovinos.

La concentración de metal en la leche (Cuadro 3) fue significativamente diferente entre las zonas $(P \leq 0.001)$, excepto para As y $\mathrm{Ni}$. El $\mathrm{Pb}$ estuvo por encima de los valores permisibles $\left(0.020 \mathrm{mg} \mathrm{kg}^{-1}\right)$ por la Comisión Europea ${ }^{(52)}$ y la WHO/FAO ${ }^{(53)}$. Sin embargo, los elementos tóxicos $\mathrm{Pb}$ y As, fueron inferiores a los valores establecidos por la Norma Oficial Mexicana NOM-184-SSA1-2002 $2^{(54)}$, que considera permisible para $\mathrm{Pb} 0.1 \mathrm{mg} \mathrm{kg}^{-1}$ y $0.2 \mathrm{mg} \mathrm{kg}^{-1}$ para As. En el caso del Cd no está reglamentado en las normas internacionales ni en la nacional.

Cuadro 3: Contenido de metales pesados en leche cruda ( $\left.\mathrm{m} \mathrm{kg}^{-1} \mathrm{MS}\right)$ por zona irrigada con aguas residuales en Puebla y Tlaxcala, México

\begin{tabular}{lccccccc}
\hline & $\mathrm{Cd}$ & $\mathrm{Pb}$ & $\mathrm{Ni}$ & $\mathrm{Cu}$ & $\mathrm{Cr}$ & $\mathrm{Zn}$ & $\mathrm{As}$ \\
\hline Zona & & & & & & & \\
1 & $0.001 \pm 0.0007^{\mathrm{b}}$ & $0.059 \pm 0.05^{\mathrm{a}}$ & $0.011 \pm 0.003^{\mathrm{a}}$ & $0.037 \pm 0.01^{\mathrm{a}}$ & $0.010 \pm 0.001^{\mathrm{b}}$ & $0.440 \pm 0.1^{\mathrm{a}}$ & $0.038 \pm 0.01^{\mathrm{a}}$ \\
2 & $0.001 \pm 0.0005^{\mathrm{b}}$ & $0.041 \pm 0.02^{\mathrm{ab}}$ & $0.012 \pm 0.005^{\mathrm{a}}$ & $0.024 \pm 0.01^{\mathrm{b}}$ & $0.016 \pm 0.007^{\mathrm{a}}$ & $0.320 \pm 0.1^{\mathrm{b}}$ & $0.029 \pm 0.01^{\mathrm{a}}$ \\
3 & $0.002 \pm 0.001^{\mathrm{ab}}$ & $0.039 \pm 0.02^{\mathrm{b}}$ & $0.012 \pm 0.005^{\mathrm{a}}$ & $0.025 \pm 0.009^{\mathrm{b}}$ & $0.015 \pm 0.009^{\mathrm{a}}$ & $0.330 \pm 0.1^{\mathrm{b}}$ & $0.033 \pm 0.02^{\mathrm{a}}$ \\
4 & $0.003 \pm 0.001^{\mathrm{a}}$ & $0.045 \pm 0.02^{\mathrm{ab}}$ & $0.012 \pm 0.004^{\mathrm{a}}$ & $0.029 \pm 0.01^{\mathrm{ab}}$ & $0.017 \pm 0.003^{\mathrm{a}}$ & $0.350 \pm 0.1^{\mathrm{b}}$ & $0.039 \pm 0.02^{\mathrm{a}}$ \\
\hline
\end{tabular}

MS=Materia seca.

${ }^{\text {ab }}$ Diferentes literales representan diferencias entre zonas $(P \leq 0.01)$.

En México, Solís et $a l^{(51)}$, informaron valores de $0.065 \mathrm{mg} \mathrm{kg}^{-1}$ para $\mathrm{Pb}$ y $0.27 \mathrm{mg} \mathrm{kg}^{-1}$ para $\mathrm{Cu}$ y $24 \mathrm{mg} \mathrm{kg}^{-1}$ para $\mathrm{Zn}$ en leche producida en áreas de riego con aguas residuales domésticas e industriales, siendo los niveles superiores a los encontrados en este estudio. Rosas et al $^{(53)}$ indican valores de As en un rango de 0.9 a $27.4 \mathrm{mg} \mathrm{kg}^{-1}$ en leche de la Comarca Lagunera en México, considerada como una región rica en arsénico, siendo estos niveles inferiores a los determinados en este trabajo. 
La determinación de los valores de biotransferencia de los metales de las plantas a la leche fue de: 1.21E-04; 3.06E-07; 2.10E-05; 8.27E-05; 1.38E-05; 2.12E-04; 1.30E-02, para AS, $\mathrm{Cd}, \mathrm{Cr}, \mathrm{Cu}, \mathrm{Ni}, \mathrm{Pb}, \mathrm{y} \mathrm{Zn}$ respectivamente, donde el As, $\mathrm{Pb}$ y Zn muestran una biotransferencia mayor y en el caso del As concuerda con los valores reportados por Rosas et al ${ }^{(55)}$.

\section{Conclusiones e implicaciones}

Se concluye que las plantas de alfalfa producida en los suelos agrícolas de la cuenca del Alto Balsas en el estado de Tlaxcala y Puebla en México, contienen metales en las partes comestibles, y el consumo de éstas por los animales representa riesgo a la salud, debido a la acumulación que se puede generar por el consumo crónico, principalmente de $\mathrm{Cd}, \mathrm{Pb}$ y As. De igual manera se ha determinado que la leche producida en estas regiones y bajo el sistema de producción de pequeños productores, contiene elementos tóxicos como el $\mathrm{Pb}$ y As que están en niveles por arriba de las normas internacionales y por debajo de las normas nacionales. Por ello es importante realizar investigaciones que ayuden a determinar el grado de riesgo, así como monitorear constantemente los suelos con el objetivo de implementar programas, para evitar la presencia de elementos tóxicos en la cadena alimenticia en las regiones analizadas, y con ello prevenir trastornos a la salud de los pobladores.

\section{Agradecimientos}

A la Asociación Universitaria Iberoamericana de Posgrado (AUIP), al Colegio de Postgraduados - Campus Puebla, al Centro Universitario de Vinculación y Transferencia de Tecnología (CUVyTT) de la Benemérita Universidad Autónoma de Puebla, por el apoyo para la realización de este trabajo. 


\section{- Literatura citada}

1. Haiyan W, Stuanes AO. Heavy metal pollution in air-water-soil-plant system of Zhuzhou city, Hunan province, China. Water Air Soil Pollut 2003;147:79-107.

2. Chang CY, Yu HY, Chen JJ, Li FB, Zhang HH, Liu CP. Accumulation of heavy metals in leaf vegetables from agricultural soils and associated potential health risks in the Pearl River Delta, South China. Environ Monit Assess 2014;186:1547-1560.

3. Bortey-sam N, Nakayama SMM, Ikenaka Y, Akoto O, Baidoo E, Beyene Y, et al. Human health risks from metals and metalloid via consumption of food animals near gold mines in Tarkwa, Ghana: Estimation of the daily intakes and target hazard quotients (THQs). Ecotoxicol Environ Saf 2015;111:160-167.

4. Sundberg J, Ersson B, Lönnerdal B, Oskarsson A. Protein binding of mercury in milk and plasma from mice and man - A comparison between methylmercury and inorganic mercury. Toxicol Appl Pharmacol 1999;137:169-184.

5. Dorea JG, Donangelo CM. Early (in uterus and infant) exposure to mercury and lead. Clin Nutr 2006;25:369-376.

6. ATSDR. The Agency for Toxic Substances and Disease Registry, Atlanta, GA. http://www.atsdr.cdc.gov/, 2013. Accessed Sept 29, 2015.

7. Gulson BL, Mizon KJ, Korsch MJ, Howarth D. Non-orebody sources are significant contributors to blood lead of some children with low to moderate lead exposure in a major lead mining community. Sci Total Environ 1996;181(3):223-230.

8. Muchuweti M, Birkett JW, Chinyanga E, Zvauya R, Scrimshaw MD, Lester JN. Heavy metal content of vegetables irrigated with mixtures of wastewater and sewage sludge in Zimbabwe: Implications for human health. Agric Ecosyst Environ 2006;112(1):41-48.

9. Duruibe JO, Ogwuegbu MOC, Egwurugwu JN. Heavy metal pollution and human biotoxic effects. Int J Phys Sci 2007;2(5):112-118.

10. Hamdi H, Benzarti S, Aoyama I, Jedidi N. Rehabilitation of degraded soils containing aged PAHs based on phytoremediation with alfalfa (Medicago sativa L.). Int Biodeterior Biodegrad 2012;67:40-47.

11. Chen F, Wang S, Mou S, Azimuddin I, Zhang D, Pan X, et al. Physiological responses and accumulation of heavy metals and arsenic of Medicago sativa $\mathrm{L}$. growing on acidic copper mine tailings in arid lands, J Geochemical Explor 2015;157:27-35.

12. Bernal Martínez LR, Rojas-Garduño MA, Vázquez-Fontes C, Espinoza-Ortega A, Estrada-Flores J, Castelán-Ortega OA. Determinación de la calidad fisicoquímica de la 
leche cruda producida en sistemas campesinos en dos regiones del Estado de México. Vet Méx 2007;38:395-407.

13. Rayas-Amor A, Estrada-Flores JG, Mould FL, Castelán-Ortega OA. Nutritional value of forage species from the Central highlands region of Mexico at different stages of maturity. C Rural, Santa María 2012;42 (4):705-712.

14. SIAP, Servicio de Información Agroalimentaria y Pesquera. Resumen nacional de ganadería. http://www.siap.gob.mx/index.php?option=com. Consultado May 29, 2016.

15. INEGI, Instituto Nacional de Estadística y Geografía. Información nacional por entidad federativa y municipios. http://www.3.inegi.org.mx/sistemas/mexicocifras/default. aspx?e=21. Consultado May 29, 2016.

16. Esmaeili A, Moore F, Keshavarzi B, Jaafarzadeh N, Kermani M. A geochemical survey of heavy metals in agricultural and background soils of the Isfahan industrial zone, Iran. Catena 2014;121:88-98.

17. Norma Oficial Mexicana NOM-021-SEMARNAT-2000. El establecimiento de las especificaciones de la fertilidad, salinidad y clasificación de suelos estudio, toma de muestras y análisis. Secretaría de Medio Ambiente y Recursos Naturales. Diario Oficial de la Federación. http://biblioteca.semarnat.gob.mx/janium/Documentos/Ciga/libros 2009/ DO2280n.pdf, 2002 Consultado Nov 12, 2015.

18. Bao Z, Wu W, Liu H, Chen H, Yin S. Impact of long-term irrigation with sewage on heavy metals in soils, crops, and groundwater. A case study in Beijing. Polish J Environ Stud 2014;23(2):309-318.

19. Wang Y, Qiao M, Liu Y, Zhu Y. Health risk assessment of heavy metals in soils and vegetables from wastewater irrigated area, Beijing-Tianjin city cluster, China. J Environ Sci 2012;24(4):690-698.

20. Amin NU, Hussain A, Alamzeb S, Begum S. Accumulation of heavy metals in edible parts of vegetables irrigated with waste water and their daily intake to adults and children, District Mardan, Pakistan. Food Chem 2013;136(3-4):1515-1523.

21. Zayed A, Gowthaman S, Terry N. Phytoaccumulation of trace elements by wetland plants: I. Duckweed. J Environ Qual 1998;27(3):715-721.

22. Ghosh M, Singh SP. A comparative study of cadmium phytoextraction by accumulator and weed species. Environ Pollut 2005;133(2):365-371. doi:10.1016/j.envpol. 2004.05.015

23. Geyer H, Scheunert I, Korte F. Bioconcentration potential of organic environmental chemicals in humans. Regul Toxicol Pharmacol 1986;6(4):313-347. 
24. Chamberlain AC. Fallout of lead and uptake by crops. Atmos Environ 1983;17(4):693706.

25. Singh A, Sharma RK, Agrawal M, Marshall FM. Health risk assessment of heavy metals via dietary intake of foodstuffs from the wastewater irrigated site of a dry tropical area of India. Food Chem Toxicol 2010;48(2):611-619. doi:10.1016/j.fct.2009.11.041.

26. Chen L, Long X-H, Zhang Z-H, Zheng X-T, Rengel Z, Liu Z-P. Cadmium accumulation and translocation in two Jerusalem artichoke (Helianthus tuberosus L.) cultivars. Pedosphere 2011;21:573-580. doi:10.1016/S1002-0160(11)60159-8.

27. Majid NM, Islam MM, Abdul Rauf R, Ahmadpour P, Abdu A. Assessment of heavy metal uptake and translocation in Dyera costulata for phytoremediation of cadmium contaminated soil. Acta Agriculturae Scandinavica Section B - Soil Plant Sci 2011;62(3):245-250.

28. Xiong C, Zhang Y, Xu X, Lu Y, Ouyang B, Ye Z, et al. Lotus roots accumulate heavy metals independently from soil in main production regions of China. Sci Hortic 2013;164:295-302.

29. Galal TM, Shehata HS. Bioaccumulation and translocation of heavy metals by Plantago major L. grown in contaminated soils under the effect of traffic pollution. Ecol Indic 2015;48:244-251.

30. SAS Institute, SAS User's Guide: Statistics Version 9.2. Statistical Analysis System Institute Cary, North Carolina, 2002; USA.

31. Stevens, Jeffrey B. Disposition of toxic metals in the agricultural food chain. Steadystate bovine milk biotransfer factors. Environ Sci Technol 1991;25:1289-1294.

32. Siebe C. Acumulación y disponibilidad de metales pesados en suelos regados con aguas residuales en el distrito de riego 03, Tula, Hidalgo, México. Rev Int Contam Ambient 1994;10:15-21. http://revistas.unam.mx/index.php/rica/article/view/30146. Consultado Nov 13, 2016.

33. Vázquez-Alarcón A, Justin-Cajuste L, Siebe-Grabach C, Alcántar-González G, De La Isla De Bauer MDL. Cadmio, níquel y plomo en agua residual, suelo y cultivos en el valle del Mezquital, Hidalgo, México. Agrociencia 2001;35:267-274.

34. CONAGUA, resúmenes mensuales de temperatura y lluvias. http://smn. conagua.gob.mx/ es/climatologia/ temperaturas-y-lluvias/resumenes-mensuales-detemperaturas-y-lluvias. Consultado Oct 12, 2016. 
35. Turer D, Maynard JB, Sansalone JJ. Heavy metal contamination in soils of urban highways: Comparison between runoff and soil concentrations at Cincinnati, Ohio. Water Air Soil Pollut 2001;132(3-4):293-314.

36. Kabata-Pendias A. Trace elements in soils and plants. Third ed. Boca Raton USA: CRC Press, Inc; 2000.

37. Peralta-Videa JR, Gardea-Torresdey JL, Walton J, Mackay WP, Duarte-Gardea M. Effects of zinc upon tolerance and heavy metal uptake in alfalfa plants (Medicago sativa). Bull Environ Contam Toxicol 2003;70(5):1036-1044.

38. Motesharezadeh B, Savaghebi-Firoozabadi GR. Bioaccumulation and phytotranslocation of nickel by Medicago sativa in a calcareous soil of Iran. Desert 2010;15(1):61-69. http://search.ebscohost.com/login.aspx?direct=true\&db=s8h\&AN= 54320222\&site=ehost-live. Accessed Oct 23, 2016.

39. Kabata-Pendias A. Agricultural problems related to excessive trace element contents of soils. Salomons W, et al. editors. Heavy metals. Springer-Verlag Berlin Heidelberg 1995:3-18. http://link.springer.com/chapter/10.1007/978-3-642-79316-5_1. Accessed Jun 23, 2016.

40. McLaughlin MJ, Smolders E, Degryse F, Rietra R. Uptake of metals from soil into vegetables. Dealing with contaminated sites. Springer 2011;325-367.

41. Peralta-Videa JR, de la Rosa G, Gonzalez JH, Gardea-Torresdey JL. Effects of the growth stage on the heavy metal tolerance of alfalfa plants. Adv Environ Res 2004;8(3):679-685.

42. Raskin I, Ensley BD. Phytoremediation of toxic metals: Using plants to clean up the environment. New York, USA: John Wiley and Sons; 2000.

43. Tu C, Ma LQ, Bondada B. Arsenic accumulation in the hyperaccumulator Chinese brake and its utilization potential for phytoremediation. J Environ Qual 1998;31(5):16711675.

44. Pinto AP, Mota AM, De Varennes A, Pinto FC. Influence of organic matter on the uptake of cadmium, zinc, copper and iron by sorghum plants. Sci Total Environ 2004; 326(1-3): 239-47. doi:10.1016/j.scitotenv.20itotenv.2004.01.004.

45. Taylor RW, Allison DW. Growth, influence of lead, cadmium, and nickel on the growth of Medicago sativa (L.). Plant Soil 1981;60(2):223-236 http://www.jstor.org/stable/ 42935329. Accessed Nov 12, 2016. 
46. Shah FR, Ahmad N, Masood KR, Peralta-Videa JR, Ahmad FD. Heavy metal toxicity in plants. M. Ashraf et al. editors. Plant adaptation and phytoremediation. Pakistán: Springer Sci Business Media B.V; 2010.

47. Wilkinson JM, Hill J, Phillips CJC. The accumulation of potentially-toxic metals by grazing ruminants. Proc Nutr Soc 2003;62(2):267-277.

48. Klaassen CD, Liu J, Diwan BA. Metallothionein protection of cadmium toxicity. Toxicol Appl Pharmacol 2009;238(3):215-220.

49. Galey FD, Slenning BD, Anderson ML, Breneman PC, Littlefield ES, Melton LA, et al. Lead concentrations in blood and milk from periparturient dairy heifers seven months after an episode of acute lead toxicosis. J Vet Diagnostic Investig 1990;2(3):222-226.

50. Canty MJ, Scanlon A, Collins DM, McGrath G, Clegg TA, Lane E, et al. Cadmium and other heavy metal concentrations in bovine kidneys in the Republic of Ireland. Sci Total Environ 2014;(485-486):223-231. doi:10.1016/j.scitotenv.2014.03.065.

51. Solis C, Isaac-Olive K, Mireles A, Vidal-Hernandez M. Determination of trace metals in cow's milk from waste water irrigated areas in Central Mexico by chemical treatment coupled to PIXE. Microchem J 2009;91(1):9-12. doi:10.1016/j.microc.2008.06.001.

52. European Commision. Commission Regulation 2006/1881/EC of 19 December 2006 replacing Regulation (EC) 466/2001 setting maximum levels for certain contaminants in foodstuffs. 2006; OJ L 364/5-24. http://eur-lex.europa.eu/legal-content/EN/ALL/ ?uri=CELEX\%3A32006R1881. Accessed Dic 20, 2016.

53. World Health Organization. Evaluation of certain food additives and contaminants. Technical Report Series no. 837. Geneva: FAO/WHO. 1993.

54. Norma Oficial Mexicana NOM-184-SSA1-2002. 2002. Productos y servicios. Leche, formula láctea y producto lácteo combinado. Especificaciones sanitarias. http://www.salud.gob.mx/unidades/cdi/nom/184ssa12.html. Consultado Jul 12, 2016.

55. Rosas I, Belmont R, Armienta A, Baez A. Arsenic concentrations in water, soil, milk and forage in Comarca Lagunera, Mexico. Water, Air, Soil Pollut 1999;112:133-149. 